\title{
DYNAMIC EQUILIBRIUM OF CLIMBING ROBOTS BASED ON STABILITY POLYHEDRON FOR GRAVITO-INERTIAL ACCELERATION
}

\author{
WARLEY F. R. RIBEIRO, KENTARO UNO and KAZUYA YOSHIDA \\ Department of Aerospace Engineering, Tohoku University, \\ Aoba 6-6-01, Aramaki-Aza, Aoba-ku, Sendai, Miyagi 980-8579, Japan \\ E-mail:warley@dc.tohoku.ac.jp, unoken@dc.tohoku.ac.jp,yoshida@astro.mech.tohoku.ac.jp \\ www.astro.mech.tohoku.ac.jp/e/ \\ KENJI NAGAOKA \\ Department of Mechanical and Control Engineering, Kyushu Institute of Technology, \\ Sensuicho 1-1, Tobata-ku, Kitakyushu, Fukuoka 804-8550, Japan \\ E-mail: nagaoka.kenji572@mail.kyutech.jp \\ www.mech.kyutech.ac.jp/srl/index_e.html
}

\begin{abstract}
Climbing robots have a high capability to explore hazardous environments, including disaster sites and extraterrestrial bodies under low-gravity. Equilibrium evaluation is necessary to plan safe locomotion for a robot, but most methods consider either only static conditions or complex friction theory. Since dynamic consideration is necessary for evaluation in low-gravity and defining friction characteristics for grippers with several contact points is challenging, we propose an equilibrium evaluation based on gravito-inertial acceleration (GIA) and stability polyhedron. We combine both static and dynamics from gravity and inertial acceleration into one single vector and calculate stability polyhedron from tumble stability theory by including the maximum holding force of a gripper. Simulations and experiments validate the method efficacy.
\end{abstract}

\section{INTRODUCTION}

Robots are a reliable and suitable solution for activities in hazardous locations, such as places affected by disasters or extraterrestrial bodies. Legged-robots are a good architecture for such applications because its locomotion system allows an easier way to surpass uneven terrains with small obstacles due to the non-continuous contact with the surface.

Robots able to climb steep terrains can be even more useful, with the increased locomotion capability to go further on the exploration of places not accessible by conventional locomotion methods, such as skylights on the surface of the Moon. ${ }^{1}$ The robot needs to attach the legs to the terrain, either relying on the static friction to prevent slippage or using a suitable grasping mechanism. Adhesion type devices to attach on smooth surfaces or grippers with several sharp spines to grasp rocky terrains are some solutions developed for climbing robots. ${ }^{2-6}$ The latter one is more suitable when we consider natural terrains in the Moon or disaster sites.

\subsection{Equilibrium Criteria}

Understanding the equilibrium of legged robots is vital for climbing robots to plan a safe motion and prevent damages on the robot due to falls. Since the beginning of the study of walking machines, researchers proposed methods to define the stability of legged robots.

The very first equilibrium criterion was based on the projection of the center of gravity on the ground surface, compared to the support polygon. ${ }^{7}$ Initially, the support polygon was defined as the polygon in which vertices were the contact points between the leg of the robot and the ground surface. The robot was considered stable if the projection of the gravity vector was inside the support polygon. 
Using the same idea as the center of gravity projection, new methods were developed to obtain the correct shape for the support polygon. By considering uneven terrains or inclined surfaces, the simple approach of taking the contact points as the vertices of the polygon is no longer valid. Friction conditions also needed to be taken into account to include the slippage phenomenon into the equilibrium evaluation. Solving the equations of static equilibrium and the non-linear inequalities for friction limits yields the support polygon, considering the center of gravity as a variable. Linear projection methods were implemented to make the computation faster but remain a relatively complex problem to solve. ${ }^{8,9}$ Moreover, no dynamics conditions were considered in those methods.

Zero-Moment Point (ZMP) is a very popular method nowadays because it considers the dynamic effects of the inertial acceleration into the equilibrium evaluation, which is required for several applications, such as biped walking. ZMP is a point in the ground which the moments in directions that tumble the robot are null. If this point is inside the support polygon, the robot is stable. ${ }^{10}$ Although ZMP is a reliable way to compute dynamic equilibrium, the computation of the support polygon still necessary.

Tumble stability is a concept based on the notion that a robot transits to an unstable condition through a tumbling motion around an axis made by two contact points. The evaluation of the direction of the tumbling moment, computed from gravitational and inertial forces, defines if the robot is or not in equilibrium. ${ }^{11}$ However, this method is based on a strong assumption that there is no slip of the legs.

Other approaches consider simple force and moment balance equations. ${ }^{12}$ Or instead of the feasibility center of gravity position, computing the feasible wrench. Solving the constraint equations that describe the friction characteristics to compute the feasible region (or polyhedron), it is possible to evaluate the robot state and plan a safe motion. ${ }^{13}$ Another proposition is to consider stable regions for the acceleration, also computed from friction and gravitational constraints. ${ }^{14}$

\subsection{Limitations of Existing Methods for Low-gravity Climbing}

In the previous section, we presented a few common methods used to characterize the state of equilibrium of a legged robot, which may not be the best approach for the case of climbing robots. Particularly if we consider robots with terrain grasping capabilities or gravity fields different from the one on Earth.

Methods that depend on friction theories to define contact forces between robot and surface are virtually incompatible for grasping-type robots. The high number of contact points, e.g., gripper with multiple spines, ${ }^{4}$ increases the number of non-linear equations to be solved. Also, in most cases, the exact number of contact points is impossible to estimate. Friction coefficients of robots grasping natural and unknown terrains might not be precisely obtained when sharp spines are attached to micro asperities of the surface.

Another issue is that most equilibrium methods consider static or quasi-static locomotion, which is sufficient for low-speed motion on Earth's gravity. When we consider lower gravitational fields, such as using legged robots to explore the Moon or small asteroids, the dynamic effects of the motion become dominant for the equilibrium analysis, and such methods must include inertial forces. The most used method that includes dynamics effects is ZMP, which requires the calculation of a support polygon. The support polygon cannot be easily obtained if the robot is on an inclined configuration relative to the gravity vector or if holding contact forces are taken into account. Considering inclined surfaces and holding forces are two essential requirements to evaluate equilibrium during climbing.

In this paper, we propose an equilibrium evaluation method based on the combination of both static and dynamic requirements into one single parameter, the gravito-inertial ac- 
celeration (GIA). We define a three-dimensional region that limits states of equilibrium for GIA, depending on the posture of the robot and the limits of the holding forces, rather than considering complex friction assumptions. Based only on the limit of holding forces, we can predict detachment of the gripper due to high pulling forces or slippage. In real-time situations, a force sensor embedded on the gripper can directly measure the holding force if the robot pulls the leg up to a limit that does not detach the gripper. Alternatively, performance evaluation tests can measure the grasping capability in various conditions beforehand, combined with a physical model of the gripper to predict attaching forces. ${ }^{5}$

In the next chapters, we present the method to calculate the three-dimensional region for stable GIA, the stability polyhedron for gravito-inertial acceleration. We define equilibrium margins based on the possible inclination and acceleration increment. Finally, we present simulation and experiment results to validate the proposed method applicability.

\section{STABILITY POLYHEDRON FOR GRAVITO-INERTIAL ACCELERATION}

From the idea of tumble stability, we define a tumbling axis $a-b$ made by two arbitrary contact points. The robot is in equilibrium about $a-b$ if the total tumbling moment $M_{a b}$ around this axis is null or is on a direction that is supported by another contact point $j$. If this condition is satisfied for all possible tumbling axes, the robot is in equilibrium. ${ }^{11}$ Tumble stability assumes that there is no slippage of the contact points, which is reasonable for climbing robots with grippers attached to the ground.

The tumbling moment $M_{a b}$ is calculated from the total moment $\overline{\boldsymbol{M}}$ and total force $\overline{\boldsymbol{F}}$ acting on the robot, as shown in eq. (1). The positions of points $a$ and $b$ are represented by $\boldsymbol{p}_{a}$ and $\boldsymbol{p}_{b}$, respectively, as shown in Fig. 1 .

$$
M_{a b}=\overline{\boldsymbol{M}} \cdot \frac{\left(\boldsymbol{p}_{a}-\boldsymbol{p}_{b}\right)}{\left|\boldsymbol{p}_{a}-\boldsymbol{p}_{b}\right|}+\overline{\boldsymbol{F}} \cdot \frac{\left(\boldsymbol{p}_{b} \times \boldsymbol{p}_{a}\right)}{\left|\boldsymbol{p}_{a}-\boldsymbol{p}_{b}\right|}
$$

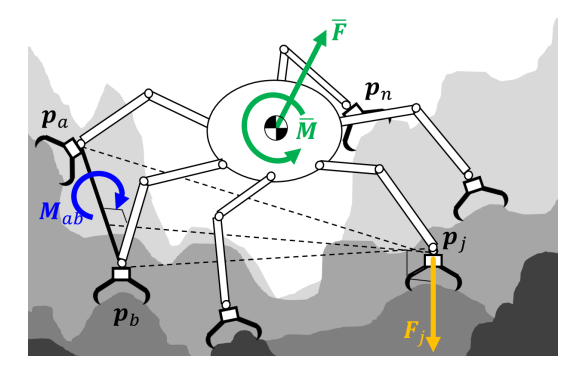

Fig. 1. Schematic overview of tumble stability concept, ${ }^{11}$ which assesses all possible moments acting around tumbling axes (line segments defined between support legs) with addition of holding forces.

Previously, our research group also included the effect of holding forces into the tumbling moment. ${ }^{15}$ A constant force $\boldsymbol{F}_{j}$ opposite to the pulling direction for the $j$-th gripper that produces a tumbling moment $M_{a b j}$, as described in the following equation:

$$
M_{a b j}=\sum_{j} \boldsymbol{F}_{j} \cdot \frac{\left(\boldsymbol{p}_{b}-\boldsymbol{p}_{j}\right) \times\left(\boldsymbol{p}_{a}-\boldsymbol{p}_{j}\right)}{\left|\boldsymbol{p}_{a}-\boldsymbol{p}_{b}\right|} .
$$

The limit for the equilibrium of a robot is defined when the total tumbling moment $M_{a b}^{\prime}=M_{a b}-M_{a b j}$ is null, representing the transition between stable and unstable states. Inside the resultant moment and forces $\overline{\boldsymbol{M}}$ and $\overline{\boldsymbol{F}}$, there is the effect of external, gravitational 
and inertial forces. As we proposed, gravitational and inertial effects can be isolated into one single gravito-inertial acceleration vector $\boldsymbol{a}_{g i}$ :

$$
\boldsymbol{a}_{g i}=\boldsymbol{g}-\boldsymbol{a}_{g}
$$

where $\boldsymbol{g}$ is the gravity vector and $\boldsymbol{a}_{g}$ is the total acceleration in the center of gravity, calculated from masses $m_{i}$ and accelerations $\boldsymbol{a}_{i}$ of all $N$ rigid links that compose the robot:

$$
\boldsymbol{a}_{g}=\frac{1}{m} \sum_{i=0}^{N} m_{i} \boldsymbol{a}_{i} .
$$

By rearranging the total tumbling moment equation in terms of the limit GIA $\boldsymbol{a}_{g i, \lim }$, we obtain eq. (5), which describes the equation of a three-dimensional plane in terms of gravito-inertial acceleration.

$$
m \boldsymbol{a}_{g i, \lim } \cdot \boldsymbol{n}_{g a b}=\sum_{j} \boldsymbol{F}_{j} \cdot\left(\boldsymbol{p}_{b}-\boldsymbol{p}_{j}\right) \times\left(\boldsymbol{p}_{a}-\boldsymbol{p}_{j}\right)+\boldsymbol{M}_{0} \cdot\left(\boldsymbol{p}_{a}-\boldsymbol{p}_{b}\right)+\boldsymbol{F}_{0} \cdot\left(\boldsymbol{p}_{b} \times \boldsymbol{p}_{a}\right) .
$$

$\boldsymbol{M}_{0}$ and $\boldsymbol{F}_{0}$ are external components of the tumbling moment, and $\boldsymbol{n}_{g a b}$ is the vector normal to the tumbling axis $a-b$ from the center of gravity position:

$$
\boldsymbol{n}_{\text {gab }}=\left(\boldsymbol{p}_{g}-\boldsymbol{p}_{a}\right) \times\left(\boldsymbol{p}_{g}-\boldsymbol{p}_{b}\right) .
$$

The plane described in eq. (5) divides the Cartesian space for accelerations into two regions: one for equilibrium states and the other for non-equilibrium states. The intersection of planes for all possible tumbling axes of a robot produces a polyhedron, which is stability polyhedron for gravito-inertial accelerations, as shown in Fig. 2.

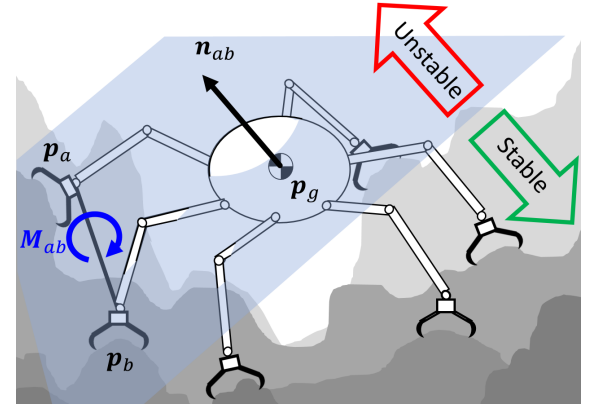

(a) Equilibrium limit plane

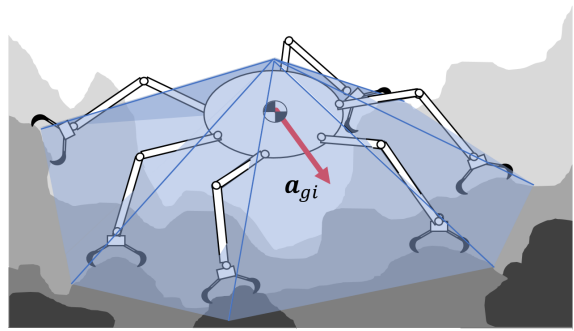

(b) Stability polyhedron

Fig. 2. Schematic overview of stability polyhedron concept. Dynamic stability of tumbling is judged based on gravito-inertial acceleration (GIA) vector inside stability polyhedron.

A mere test if the gravito-inertial acceleration vector is inside or not stability polyhedron is sufficient to evaluate the equilibrium state of a climbing robot. The equilibrium examination is a fast process, due to the facility to compute the shape of stability polyhedron, by using linear equations that depend only on the posture of the robot and the maximum holding force of the grippers.

\subsection{Acceleration Margin and Inclination Margin}

Quantitative evaluation of the degree of equilibrium of the robot is helpful to compare two different states and decide if the robot is approaching unstable conditions. Motion controllers can benefit from such numerical parameters of stability to generate a safe motion. 
Our first proposition for quantitative analysis is the inclination margin for gravito-inertial acceleration, which is the angle between the GIA vector and the limit plane for a tumbling axis. The minimum value among all tumbling axes is the inclination margin $\theta_{\text {marg: }}$ :

$$
\theta_{\text {marg }}=\min \left(\cos ^{-1}\left(\frac{\boldsymbol{n}_{g a b} \cdot \boldsymbol{a}_{g i}}{\left|\boldsymbol{n}_{g a b}\right|\left|\boldsymbol{a}_{g i}\right|}\right)-\cos ^{-1}\left(\frac{\left|\boldsymbol{a}_{g i, \lim }\right|}{\left|\boldsymbol{a}_{g i}\right|}\right)\right) .
$$

The inclination margin represents how much a robot in a certain configuration could rotate and still be in equilibrium if there are no changes in the inertial acceleration. This means that the robot can analyze in advance required postures for given static scenarios or what are the reachable limits of inclinations for a surface to be explored.

Another proposed parameter is the gravito-inertial acceleration margin, defined by the acceleration magnitude increment for GIA along the direction normal to the tumbling axis necessary to reach the limiting plane. As for the inclination margin, the minimum value among all tumbling axes is the acceleration margin $a_{\text {marg }}$ :

$$
a_{\mathrm{marg}}=\min \left(\left|\boldsymbol{a}_{g i, \lim }\right|-\frac{\boldsymbol{n}_{g a b} \cdot \boldsymbol{a}_{g i}}{\left|\boldsymbol{n}_{g a b}\right|}\right) .
$$

The acceleration margin represents the maximum acceleration increment that can be applied in any direction that does not cause the robot to tumble. This is useful for a quick check during the motion phase of the robot if the next step to be made is safe or not.

\section{SIMULATION}

Simulations of climbing robots are useful to test stability polyhedron for gravito-inertial acceleration since we can change postures of the robot, dynamic parameters, and gravity fields without restrictions. In this section, we present examples of stability polyhedrons for static and dynamic cases.

We consider the quadruped robot with passive-type grippers developed by our group as a model for the climbing robot, composed by rigid links connected by a total of 12 rotational joints -3 for each leg. The robot is placed with three legs grasping a fractal surface created to simulate natural rocky terrains, in an environment with lunar gravity $g=1.62 \mathrm{~m} / \mathrm{s}^{2}$. The base is set $0.14 \mathrm{~m}$ above the surface and legs are set with distances of $0.12 \mathrm{~m}$ in the horizontal plane relative to the base center. Grippers have a holding force of $3.2 \mathrm{~N}$. We implemented a set of functions called equ_gia to generate the simulations, where more details of the calculations are described. ${ }^{a}$

First, for a static case, we apply stability polyhedron for GIA, as shown by Fig. 3. Stability polyhedron, drawn in blue, is composed of three planes because there are three possible tumbling axes. The GIA vector shown in red in the figure, coinciding with the gravity vector, is inside the polyhedron, indicating that the robot is in equilibrium. The cross-section of a plane through the GIA vector along $y-z$ directions is shown inside the magenta box for better comparison between vector and polyhedron. The calculated acceleration margin of $2.16 \mathrm{~m} / \mathrm{s}^{2}$ and inclination margin of $180^{\circ}$ also confirm the stable state of the robot.

Second, we consider a dynamic case, where the same robot in the same conditions now has its base accelerated in the horizontal $y$-direction by $7 \mathrm{~m} / \mathrm{s}^{2}$. Stability polyhedron does not change since it depends only on the robot's posture and the holding forces. However, the GIA vector does not coincide with the gravity vector, which is shown in green in Fig. 4 for the results of the dynamic case simulation. Since the GIA vector is outside stability polyhedron, as shown in the cross-section, this represents an unstable case.

$\overline{{ }^{a} \text { https://github.com/ribeirowarley/equ_gia }}$ 

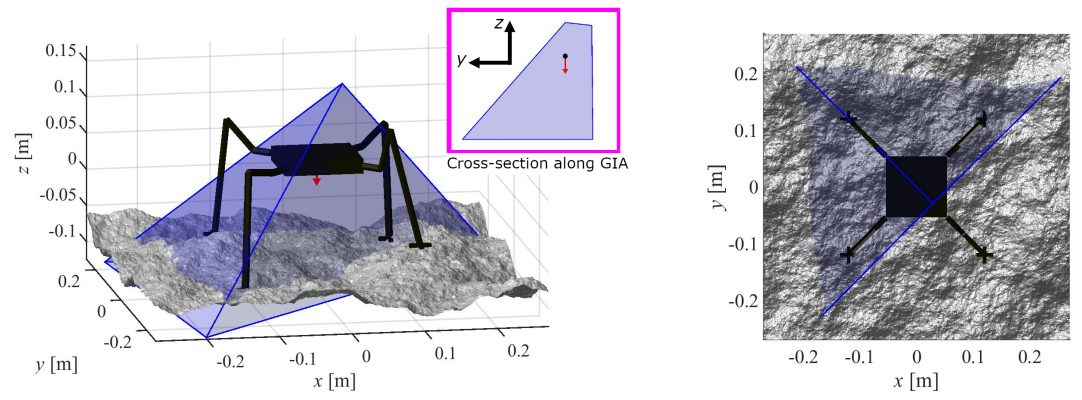

Fig. 3. Results for static case simulation.
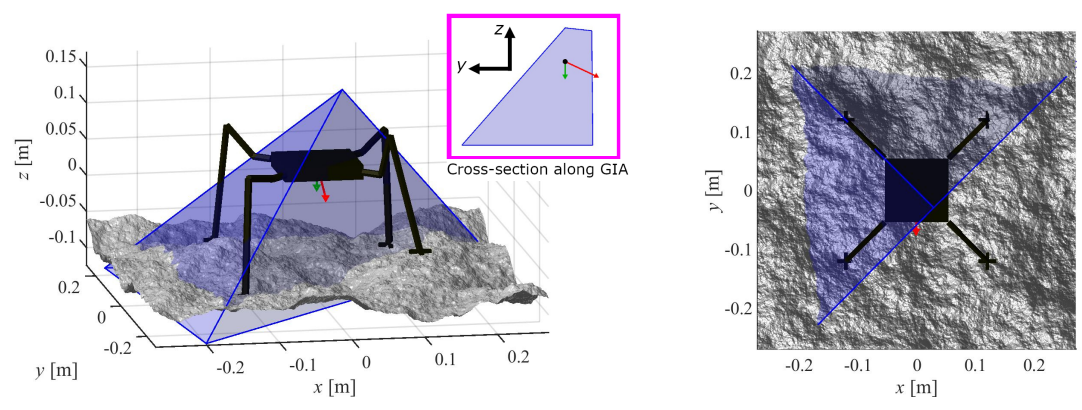

Fig. 4. Results for dynamic case simulation.

\section{EXPERIMENT}

We also performed experiments with a real robot to validate stability polyhedron for GIA as an equilibrium criterion. Due to the difficulty of performing dynamic tests in reduced gravity with our current devices, a simple setup with a static robot under Earth's gravity field is considered. To verify the proposed method, both stable and unstable conditions are needed. Therefore, we tested different inclinations for the same posture of the robot.

\subsection{Method}

The robot is a quadruped robot with servo-motors to actuate rotational joints and doublespine passive grippers. ${ }^{5}$ Pulling experiments for the gripper showed an average holding force of $6.8 \mathrm{~N}$, and a minimum holding force of $3.2 \mathrm{~N}$. The simulated terrain is composed of a wooden plate with a changeable inclination angle measured by a protractor. Grasping targets are holders with conic shapes covered with sandpaper to emulate the roughness of natural rocks. These holders have the same opening angle and sandpaper type as the ones used for the pulling experiments to evaluate the gripper performance.

The posture of the robot is defined similarly as the simulations, with a base height of $0.14 \mathrm{~m}$ and legs set with distances of $0.12 \mathrm{~m}$ in the horizontal plane. The procedure for the experiment is described below, with Fig. 5 showing one example of an equilibrium test with the approximate GIA vector and the correspondent stability polyhedron. The figure also shows a simulation with the calculated stability polyhedrons, for both minimum (orange) and average (green) holding forces. The example presents the enlargement of the polyhedron for larger forces.

(1) Set a fixed inclination for the wooden plate, beginning at 50 degrees

(2) Place the robot as the grippers can hold at the grasping targets and check if the robot is in equilibrium or if it tumbles 
(3) Repeat (2) five times for the same inclination of the wooden plate

(4) Update the inclination by a 5 degrees increment

(5) Repeat from (2) until approximately 80 degrees

(6) Finally, compare with stability polyhedron for GIA.

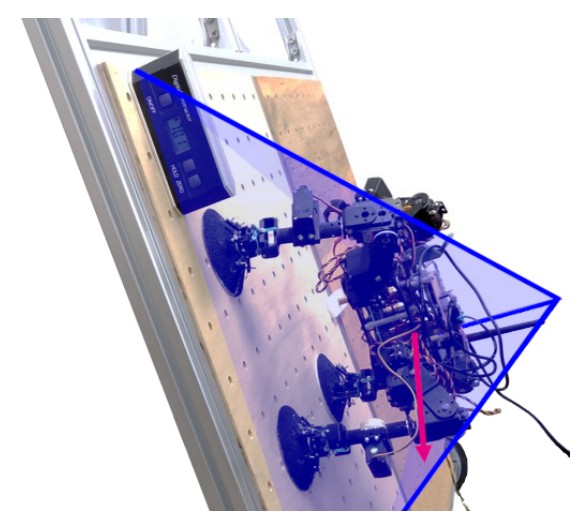

(a) Experiment

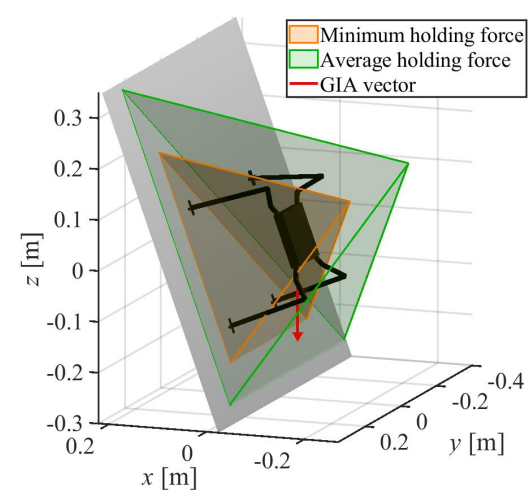

(b) Simulation

Fig. 5. (a) overview of static experiment using the robot test-bed developed in our research group ${ }^{5}$ with drawings of stability polyhedron and GIA vector. (b) simulation for experiment setup with stability polyhedrons for minimum (orange) and average (green) holding forces.

\subsection{Results and Discussion}

Fig. 6 summarizes the results for the static experiments, showing the blue dots for the grasping success rate percentage of each tested inclination angle. Dotted lines represent the expected success rate for a robot with the same configuration based on stability polyhedron for GIA, for both the minimum (orange) and average (green) values for holding force.

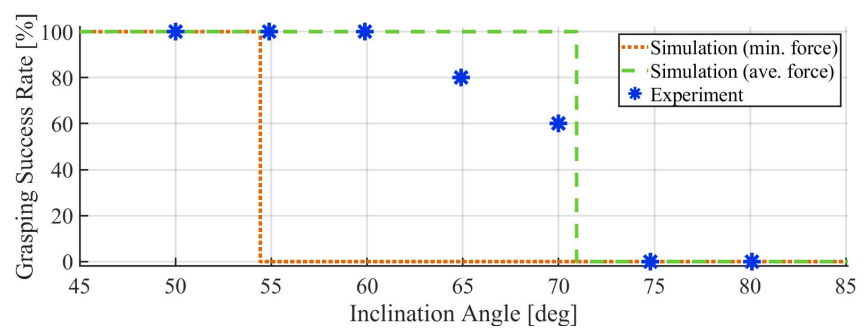

Fig. 6. Comparison of grasping success rate between experimental results and simulations for GIA stability polyhedron.

The graph shows that stability polyhedron can be used to verify if a robot is in equilibrium given the holding force, the posture of the robot and gravito-inertial acceleration. The experiment results show that the robot is in equilibrium for $100 \%$ of cases if the inclination angle is smaller than $54.4^{\circ}$, as predicted for the minimum value for holding force of $3.2 \mathrm{~N}$. By extrapolating experimental results, the robot is in equilibrium in approximately $50 \%$ of cases for the inclination of $70.9^{\circ}$, based on the average holding force value of $6.8 \mathrm{~N}$.

The real value of a holding force is variable with time and is highly dependent on the gripping conditions, such as the grasping target shape, roughness, material, and gripper performance. Knowing the exact force is nearly impossible and using a minimum value 
of holding forces for real applications could be a more suitable solution, although it is a conservative consideration.

\section{CONCLUSION}

In this paper, we describe a method to evaluate the dynamic equilibrium of climbing robots based on stability polyhedron for the gravito-inertial acceleration vector, which includes both the gravity and inertial accelerations. We consider the stability relative to the tumbling motion for robots based on the holding capabilities of the grippers. This method can be used for any type of surface, gravitational field and does not require high computational time due to nonlinear friction characteristics.

Stability polyhedron for gravito-inertial accelerations is particularly useful for natural environments climbing robots with grippers since the exact friction conditions are nearly impossible to measure in real-time. The simple use of a maximum holding force allows fast and reliable equilibrium evaluation, as verified by the performed simulations and experiments.

\section{Acknowledgments}

This work is partly supported by JSPS KAKENHI Grant Number 19J20685.

\section{References}

1. T. Kaku, J. Haruyama, W. Miyake, A. Kumamoto, K. Ishiyama, T. Nishibori, K. Yamamoto, S. T. Crites, T. Michikami, Y. Yokota, R. Sood, H. J. Melosh, L. Chappaz and K. C. Howell, Geophysical Research Letters 44, 10,155 (2017).

2. A. Asbeck, S. Dastoor, A. Parness, L. Fullerton, N. Esparza, D. Soto, B. Heyneman and M. Cutkosky, Climbing rough vertical surfaces with hierarchical directional adhesion, in Proc. IEEE Int. Conference on Robotics and Automation, (Kobe, Japan, 2009).

3. P. Glick, S. A. Suresh, D. Ruffatto, M. Cutkosky, M. T. Tolley and A. Parness, IEEE Robotics and Automation Letters 3, 903 (2018).

4. A. Parness, N. Abcouwer, C. Fuller, N. Wiltsie, J. Nash and B. Kennedy, LEMUR 3: A limbed climbing robot for extreme terrain mobility in space, in Proc. IEEE Int. Conference on Robotics and Automation, (Singapore, Singapore, 2017).

5. K. Nagaoka, H. Minote, K. Maruya, Y. Shirai, K. Yoshida, T. Hakamada, H. Sawada and T. Kubota, IEEE Robotics and Automation Letters 3, 1765 (2018).

6. M. J. Spenko, G. C. Haynes, J. A. Saunders, M. R. Cutkosky, A. A. Rizzi, R. J. Full and D. E. Koditschek, Journal of Field Robotics 25, 223 (2008).

7. R. McGhee and A. Frank, Mathematical Biosciences 3, 331 (1968).

8. T. Bretl and S. Lall, IEEE Transactions on Robotics 24, 794 (2008).

9. A. Del Prete, S. Tonneau and N. Mansard, Fast algorithms to test robust static equilibrium for legged robots, in Proc. IEEE Int. Conference on Robotics and Automation, (Stockholm, Sweden, 2016).

10. M. Vukobratović and B. Borovac, International Journal of Humanoid Robotics 01, 157 (2004).

11. K. Yoneda and S. Hirose, Tumble stability criterion of integrated locomotion and manipulation, in Proc. IEEE/RSJ Int. Conference on Intelligent Robots and Systems, (Osaka, Japan, 1996).

12. A. Shimada and Nguyen Anh Dung, Equilibrium state control for climbing robot, in Proc. IEEE 14th Int. Workshop on Advanced Motion Control, (Auckland, New Zealand, 2016).

13. R. Orsolino, M. Focchi, C. Mastalli, H. Dai, D. G. Caldwell and C. Semini, IEEE Robotics and Automation Letters 3, 3363 (2018).

14. S. Caron and A. Kheddar, Multi-contact walking pattern generation based on model preview control of 3D COM accelerations, in Proc. IEEE-RAS 16th Int. Conference on Humanoid Robots, (Cancun, Mexico, 2016).

15. K. Uno, W. F. R. Ribeiro, W. Jones, Y. Shirai, H. Minote, K. Nagaoka and K. Yoshida, Gait Planning for a Free-Climbing Robot Based on Tumble Stability, in Proc. IEEE/SICE Int. Symposium on System Integration, (Paris, France, 2019). 